\title{
Privatizar ou não privatizar? Uma análise longitudinal dos serviços de abastecimento de água no Brasil
}

\author{
To privatize or not to privatize? A longitudinal \\ analysis of the Brazilian water supply services
}

Lorena Soares Laia Cabral', Erica Castilho Rodrigues ${ }^{2}$, Alberto Fonseca ${ }^{3 *}$

口-

\begin{abstract}
RESUMO
A privatização é frequentemente mencionada como uma potencial solução para os problemas dos serviços de abastecimento de água. Todavia, são raros os estudos sobre os reais impactos da privatização, sobretudo no Brasil. O objetivo deste trabalho foi comparar o desempenho dos prestadores públicos e privados no Brasil entre 2003 e 2013. A metodologia baseou-se na análise dos indicadores do Sistema Nacional de Informações sobre Saneamento, por meio de análises fatoriais e testes não paramétricos. Também testou-se a aplicação de modelos de regressão linear, embora sem sucesso. A análise visual dos boxplots sugeriram diferenças no desempenho entre prestadores público e privado em anos específicos. Essa situação foi confirmada por meio dos testes não paramétricos. Todavia, os serviços privatizados já tinham o melhor desempenho desde o início do período de análise, o que corrobora o argumento de que a privatização tende a ocorrer em municípios que já apresentam condições favoráveis de abastecimento deágua. Estudos futuros são, finalmente, sugeridos.

Palavras-chave: serviços de abastecimento de água; privatização; Sistema Nacional de Informação sobre Saneamento Ambiental; indicadores; Brasil.
\end{abstract}

\begin{abstract}
Privatization is often mentioned as a potential solution to water supply problems. However, few studies have addressed the real impacts of privatization, particularly in Brazil. The objective of this study was to compare the performance of public and private water supply services in Brazil between 2003 and 2013. The methodology was based on the analysis of the indicators of the Brazilian National Information System on Sanitation, through factor analyses and nonparametric tests. The study also conducted linear regression models, without success. The visual analysis of the boxplots suggested significant differences in the overall performance of public and private groups in specific years. This situation was confirmed in nonparametric tests. However, privatized services had the best performance since the beginning of the analysis period, a fact that corroborates the argument that privatization tends to occur in municipalities with more favorable water supply services. Future studies are, finally, suggested.
\end{abstract}

Keywords: water supply services; privatization; National Environmental Sanitation Information System; indicators; Brazil.

\section{INTRODUÇÃO}

Durante a década de 1990, houve uma intensa promoção da privatização dos serviços de água e esgoto de países em desenvolvimento. O propósito era estimular o desenvolvimento desse setor em seus territórios, atraindo novas fontes de investimentos associadas ao ganho de eficiência e qualidade desses serviços (ARARAL, 2009; BUDDS \& MCGRANAHAN, 2003; PÉRARD, 2009; VARGAS \& GOUVELLO, 2011).

Instituições públicas e privadas possuem diferentes abordagens de gestão organizacional e orçamentária, uma situação que pode influenciar o desempenho dos serviços de abastecimento de água. Teoricamente, os serviços públicos tendem a atingir mais facilmente os objetivos de natureza social, mas com diversos riscos, como de interferências políticas. Os operadores privados possuem uma visão empresarial, podendo dar maior eficiência aos seus processos operacionais a fim de maximizar o lucro (BORRAZ; PAMPILLON; OLARREAGA, 2013). Na literatura, há estudos, nacionais e internacionais, que relatam melhorias alcançadas pela privatização, bem como estudos que desmitificam a superioridade do desempenho das entidades privadas em relação às entidades públicas (ZAKI \& NURUL AMIN, 2009; BAER, 2014; HAILU; OSORIO; TSUKADA, 2012; OUDA; AL-WAKED; ALSHEHRI, 2014; $\square$

'Programa de Pós-graduação em Engenharia Ambiental da Universidade Federal de Ouro Preto (UFOP) - Ouro Preto (MG), Brasil. 2Professora do Departamento de Estatístca da UFOP - Ouro Preto (MG), Brasil.

3Professor do Departamento de Engenharia Ambiental da UFOP - Ouro Preto (MG), Brasil.

*Autor correspondente: albertof@em.ufop.br

Recebido: 06/07/2016 - Aceito: 06/06/2017 - Reg. ABES: 166181 
BUDDS \& MCGRANAHAM, 2003; ARARAL, 2009; PÉRARD, 2009; FARIA; FARIA; MOREIRA, 2005; VARGAS \& GOUVELLO, 2011 ).

De acordo com Budds e McGranaham (2003) e Araral (2009), as empresas privadas investem em países com renda média, portanto, não contribuem para a ampliação do acesso à água potável em países mais pobres. Ainda segundo Araral (2009), as fontes de financiamento são as mesmas das instituições públicas, e a eficiência pode estar ligada a outros fatores que não a gestão ser privada ou pública, visto que há bons exemplos em ambos os casos. Pérard (2009) também afirma que a simples participação privada no abastecimento de água não significa resultados positivos na eficiência.

No Brasil, o incentivo à participação privada foi um dos programas federais implantados após o fim do Plano Nacional de Saneamento (PLANASA). Porém, não atingiu um público tão significativo quanto nos países vizinhos, permeando em torno de $10 \%$ da população brasileira (FOSTER, 2005; VARGAS \& GOUVELLO, 2011; SAIANI; TONETO JÚNIOR, 2010; SCRIPTORE \& TONETO JÚNIOR, 2012). Essa situação permanece pouco alterada, visto que as concessionárias privadas, em 2015, atuavam em apenas 304 (i.e., 5,4\%) dos 5.570 municípios brasileiros (ABCON \& SINDCON, 2015).

Atualmente, o cenário brasileiro de provisão dos serviços de água e esgoto é constituído por diversos modelos de prestadores com gestão pública e gestão privada: autarquias, empresas públicas, sociedade de economia mista, administração direta municipal, empresa privada e organização social (BRASIL, 2014). As principais diferenças entre esses modelos se concentram na gestão e na composição do capital.

Os efeitos da gestão privada no desempenho ao longo do tempo nos serviços de água ainda foram pouco explorados no cenário brasileiro. Recentemente, pesquisas compararam esses diferentes modelos de prestadores e indicaram que não há um modelo com um desempenho satisfatório em todos os aspectos - operacional, financeiro e social que envolve o abastecimento de água (DA SILVA E SOUZA; DE FARIA; MOREIRA, 2007; FARIA; FARIA; MOREIRA, 2005; FERRO et al., 2014; HELLER; HELLER; HELLER, 2009; HELLER et al., 2012; OLIVEIRA; REZENDE; HELLER, 2011; SCRIPTORE; TONETO JÚNIOR, 2012; PALUDO \& BORBA, 2013; SABBIONI, 2008; SAIANI \& TONETO JÚNIOR, 2010; TUPPER \& RESENDE, 2004). De maneira geral, esses estudos mantiveram um foco em determinado período, recorte geográfico e aspectos dos serviços. Além disso, nenhum deles investigou o panorama nacional numa escala longitudinal e, principalmente, a influência da privatização no desempenho dos prestadores.

Uma importante fonte de dados que pode ser utilizada para esclarecer a performance dos prestadores é o Sistema Nacional de Informação sobre Saneamento (SNIS), que fornece informações e indicadores dos prestadores de serviços de água e esgoto atuantes no Brasil desde o ano de referência 1995. Ao todo, são 83 indicadores divididos nas seguintes famílias: Econômico-financeiro e administrativo, Operacional,
Contábeis e Qualidade (BRASIL, 2014). Todavia, o potencial do SNIS para estudos longitudinais sobre os efeitos do modelo de participação privada parece ainda pouco explorado pela academia brasileira.

Este trabalho teve como objetivo realizar uma análise comparativa do desempenho dos prestadores públicos e privados ao longo de 11 anos - 2003 a 2013 - com base na amostra de indicadores do SNIS para os serviços de água. Os resultados apresentados a seguir ajudam na compreensão dos efeitos da participação privada na provisão dos serviços de abastecimento de água no Brasil, um conhecimento que pode ser útil não apenas para pesquisadores, mas também para gestores do setor de saneamento do Brasil e do mundo.

\section{METODOLOGIA}

\section{Banco de dados}

Para a definição da composição dos grupos, público e privado, considerou-se a natureza jurídica dos prestadores, conforme a definição de MCIDADES (BRASIL, 2014). O grupo público foi composto por administração pública direta, autarquia, sociedade de economia mista com gestão pública e empresa pública; enquanto o grupo privado foi formado pelas empresas privadas e pelas sociedades de economia mista com gestão privada. Neste trabalho, só foram analisados os prestadores de serviços de água que se mantiveram ativos em todos os anos analisados — de 2003 a 2013 - e que não sofreram alteração da sua natureza jurídica. Além disso, foram removidos da base de dados os municípios que tinham dois prestadores, sendo um público e outro privado.

Obteve-se um total de 3.386 prestadores de serviços de água no grupo público e 398 no grupo privado. Tal quantidade é proporcional à quantidade de municípios, visto que cada prestador corresponde a apenas um município. Contudo, aproximadamente 94\% (337 municípios) do grupo privado era representado por um mesmo prestador, cuja natureza jurídica é sociedade de economia mista com gestão privada (Sanepar). Para verificar se a predominância desse prestador específico no grupo privado influenciaria nos resultados, foram realizadas as análises estatísticas considerando o grupo privado com e sem a Sanepar. Ou seja, foram consideradas, inicialmente, como grupo privado empresa privada e sociedade de economia mista com gestão privada; depois, foram realizadas as mesmas análises, considerando apenas empresa privada como grupo privado, e foi criado outro grupo, Sanepar, com apenas a sociedade de economia mista com gestão privada.

Os dados dos indicadores de desempenho dos serviços de água foram obtidos por meio do aplicativo "Série Histórica do SNIS", para anos de referência de 2003 a 2013, constituindo uma série histórica de 11 anos (BRASIL, 2015). A base de dados foi composta de 43 indicadores, os quais foram mensurados para todos os anos analisados e cujas fórmulas de cálculo não sofreram alteração durante esse período. Além disso, 
foram obtidos dados sobre população total, Produto Interno Bruto (PIB) e PIB per capita do Instituto Brasileiro de Geografia e Estatística (IBGE).

\section{Seleção dos indicadores do Sistema Nacional de Informação sobre Saneamento e criação de índice agregado}

A análise fatorial foi utilizada a fim de reduzir o número de indicadores do SNIS e resumir as características em comum em fatores com a mínima perda de informação (ALENCAR FILHO \& ABREU, 2005; BEZERRA \& CORRAR, 2006; FIGUEIREDO FILHO \& SILVA JÚNIOR, 2010; HAIR et al., 2006). A análise fatorial é uma técnica útil na criação de índices agregados, conforme proposto em Bollmann e Marques (2000), Rezende, Fernandes e Silva (2007) e Silveira, Silva e Carvalho (2008). A execução dessa etapa seguiu três estágios:

1. verificação da adequabilidade da base de dados ao modelo fatorial;

2. determinação da técnica de extração e do número de fatores a serem extraídos;

3. decisão do tipo de rotação dos fatores (FIGUEIREDO FILHO \& SILVA JÚNIOR, 2010).

Em relação à adequabilidade da base de dados, observou-se o padrão de correlação entre os indicadores por meio da matriz de correlação, destacando os coeficientes de correlação superiores a 0,3. Além disso, aplicou-se o critério Kaiser-Meyer-Olkin (KMO) e o teste de esfericidade de Bartelett. A aplicação da análise fatorial é considerada adequada quando o valor do critério KMO for igual ou maior a 0,5 e o teste de Bartelett rejeitar a hipótese nula (H0) de que a matriz de correlação é uma matriz identidade a um nível de significância de 5\% (HAIR et al., 2006).

Em seguida, definiu-se a quantidade de fatores a serem extraídos por meio do critério de Kaiser, o qual considera como significantes apenas os fatores que possuem autovalores maiores que 1 (HAIR et al., 2006). O método de extração dos fatores utilizado foi o de mínimos quadrados (REVELLE, 2015; HARMAN \& JONES, 1966). Aplicou-se, também, a rotação ortogonal VARIMAX, visto que ela faz com que cada variável tenha uma carga fatorial elevada em apenas um fator (COSTELLO \& OSBORNE, 2005; FIGUEIREDO FILHO \& SILVA JÚNIOR, 2010).

A capacidade dos fatores em expressar a variabilidade das variáveis observadas (indicadores do SNIS) foi avaliada por meio da comunalidade. Ela representa a parcela da variância da variável observada que pode ser explicada pelos fatores comuns, e seu valor pode variar de 0 a 1 , sendo que o valor mínimo aceitável é 0,5 (HAIR et al., 2006).

A interpretação dos fatores foi feita com base no valor das cargas fatoriais, identificando a variável que exerce maior influência naquele fator. Quanto maior o seu valor, maior é a capacidade do fator em expressar a característica medida por essa variável. Dessa forma, neste trabalho, considerou-se como carga significativa aquela que foi igual ou superior a 0,4 . As variáveis que apresentaram a carga fatorial significativa em mais de um fator (carga cruzada) foram eliminadas. Em casos de eliminação, todas as etapas anteriores foram repetidas e uma nova matriz fatorial foi gerada e reavaliada. Isso foi feito até se obter um conjunto de fatores interpretáveis e que representassem a variabilidade comum dos indicadores.

Os escores foram calculados para cada indivíduo com base nas cargas fatoriais de todas as variáveis no fator, utilizando o método de regressão. Os valores dos escores fatoriais foram utilizados nas etapas posteriores deste trabalho, a fim de comparar o desempenho dos prestadores de serviços de água. Devido à diferença na escala dos indicadores, foi necessário normalizar os dados dos escores para que os seus valores estivessem em uma unidade adimensional comum, que variasse de 0 a 1 . Para tanto, foi utilizada a metodologia de transformação linear pela regra do intervalo de valor, descrita em Figueirêdo et al. (2010). Portanto, quanto mais próximo de 1 , melhor é o desempenho do prestador naquele índice; consequentemente, quanto mais próximo de 0 , pior é o seu desempenho.

\section{Testes não paramétricos e análise de regressão}

Inicialmente, foram construídos gráficos boxplots e realizado o teste Shapiro-Wilk para avaliar a normalidade dos dados. Após a confirmação da não normalidade dos dados, foram aplicados dois testes não paramétricos para inferir se havia diferença estatisticamente significante entre os grupos em cada índice, considerando ano a ano, separadamente. O teste de Wilcoxon-Mann-Whitney foi aplicado para comparar os grupos público e privado, e o teste de Kruskal-Wallis, os grupos público, privado e Sanepar. Nesse último caso, foi necessário aplicar um teste de comparações múltiplas par a par para identificar em quais grupos havia diferença.

Em seguida, a pesquisa buscou realizar uma análise de regressão linear múltipla, de modo a revelar padrões de interação e associação nos dados, mostrando, potencialmente, qualquer evidência de causa e efeito entre a privatização e o desempenho dos serviços. Por meio desses procedimentos, pode-se verificar quais variáveis explicativas estão relacionadas com a variável resposta, controlando-se outras variáveis relevantes. A estimativa dos parâmetros fornece a importância de cada variável no modelo (DRAPER \& SMITH, 1998).

Ajustou-se um modelo de regressão linear para cada índice obtido na análise fatorial, no qual a variável resposta (dependente) foi o próprio índice. As variáveis explicativas (independentes) foram: população total, PIB e PIB per capita, representando as variáveis socioeconômicas municipais; uma variável categórica que indica se o prestador é público, privado ou Sanepar; e o ano de referência, que também entrou no modelo como uma variável categórica. As variáveis socioeconômicas (população total, PIB, PIB per capita) dos municípios foram incluídas para garantir uma comparação entre prestadores sujeitos a relações aproximadamente homogêneas.

A estimativa do parâmetro associado à variável categórica permite verificar se existe diferença na média dos indicadores entre os grupos, controlando pelas demais variáveis presentes no modelo. Os parâmetros associados ao ano de referência permitem identificar tendências temporais 
na evolução dos indicadores. A interação entre essas duas variáveis (ano e grupo) permite comparar a evolução dos grupos (público, privado e Sanepar).

O modelo de regressão linear múltipla é um modelo simples, mas que tem uma série de pressupostos que precisam ser atendidos. Entre os principais, estão: independência dos erros, a normalidade $\mathrm{N}\left(0, \sigma^{2}\right)$, linearidade e homocedasticidade (HAIR et al., 2006). Esses pressupostos são avaliados por meio da análise do resíduo que é estimativa do erro do modelo. O resíduo é obtido a partir da diferença entre o valor observado e o valor ajustado pelo modelo (FIGUEIREDO FILHO et al., 2011). Essa análise pode ser feita por meio de gráficos de probabilidade normal e do gráfico de resíduos versus valores ajustados, que juntos permitem avaliar todos os pressupostos listados anteriormente. Todas as análises estatísticas foram realizadas no software estatístico R.

\section{RESULTADOS E DISCUSSÃO}

\section{Seleção de indicadores e construção de índices via análise fatorial}

$\mathrm{O}$ índice KMO e o teste de esfericidade de Bartlett indicaram a adequação da aplicação da análise fatorial para a amostra inicial de indicadores do SNIS. No entanto, algumas variáveis apresentaram cargas cruzadas (indeterminação fatorial) ou comunalidade inferior a 0,5. Diante disso, elas foram eliminadas e todas as etapas foram repetidas. O conjunto de variáveis que se ajustaram bem ao modelo fatorial e que geraram fatores interpretáveis foi:

- IN010 - Índice de micromedição relativo ao volume disponibilizado (\%);

- IN013 - Índice de perdas de faturamento (\%);

- $\quad$ IN028 - Índice de faturamento de água (\%);

- IN079 - Índice de conformidade da quantidade de amostras cloro residual (\%);

- $\quad$ IN080 - Índice de conformidade da quantidade de amostras turbidez (\%);

- IN085 - Índice de conformidade da quantidade de amostras coliformes totais (\%);

- IN007 - Incidência da despesa de pessoal e de serviços de terceiros nas despesas totais com os serviços (\%);

- IN035 - Participação da despesa com pessoal próprio nas despesas de exploração (\%);

- IN036 - Participação da despesa com pessoal total (equivalente) nas despesas de exploração (\%).

A definição de cada indicador pode ser consultada em MCIDADES (BRASIL, 2014).

Os coeficientes de correlação entre esses indicadores apresentaram, em sua maioria, valores acima de 0,3. O valor do índice KMO foi 0,77 e o teste de esfericidade de Bartlett rejeitou a H0, visto que o valor $\mathrm{p}$ foi igual a 0 . Tais resultados mostraram que a aplicação da análise fatorial para esses indicadores é adequada (FIGUEIREDO FILHO \& SILVA JÚNIOR, 2010; HAIR et al., 2006).

A partir disso, foram retidos três fatores utilizando o critério de Kaiser, visto que apenas eles apresentaram autovalores maiores que 1 , conforme Tabela 1. Além disso, esses três fatores explicaram $89 \%$ da variabilidade dos dados, o que está acima do mínimo recomendado pela literatura, 60\% (FIGUEIREDO FILHO \& SILVA JÚNIOR, 2010; HAIR et al., 2006).

A Tabela 2 apresenta os valores das cargas fatoriais rotacionadas pelo método ortogonal VARIMAX, assim como as respectivas comunalidades de cada variável. Observa-se que a comunalidade de cada variável variou de 0,62 a 1, ou seja, mais de $62 \%$ da variância dos indicadores foi explicada pelo conjunto de fatores, o que confirma que os dados são adequados para a aplicação do modelo fatorial. As características comuns desses nove indicadores foram resumidas em três fatores, que foram interpretados como novos índices nas etapas seguintes.

As variáveis com maiores cargas no fator 1 foram: IN010 Índice de micromedição relativo ao volume disponibilizado (\%), IN013 Índice de perdas faturamento (\%) e IN028 Índice de faturamento de água

Tabela 1 - Autovalores e variância acumulada dos fatores.

\begin{tabular}{c|c|c|c} 
Fator & Autovalores & \% variância & \% acumulada \\
\hline 1 & 4,14 & 45,96 & 45,96 \\
\hline 2 & 2,59 & 28,75 & 74,71 \\
\hline 3 & 1,37 & 15,26 & 89,97 \\
\hline 4 & 0,28 & 3,08 & 93,05 \\
\hline 5 & 0,23 & 2,52 & 95,57 \\
\hline 6 & 0,16 & 1,81 & 97,38 \\
\hline 7 & 0,13 & 1,44 & 98,82 \\
\hline 8 & 0,11 & 1,18 & 100,00 \\
\hline 9 & 0,00 & 0,00 & 100,00 \\
\hline
\end{tabular}

Tabela 2 - Matriz de cargas fatoriais e comunalidades das variáveis.

\begin{tabular}{c|c|c|c|c} 
& Fator 1 & Fator 2 & Fator 3 & Comunalidade \\
\hline IN010 & 0,78 & 0,31 & 0,03 & 0,71 \\
\hline IN013 & $-0,99$ & $-0,14$ & $-0,01$ & 1 \\
\hline IN028 & 0,99 & 0,14 & 0,01 & 1 \\
\hline IN079 & 0,16 & 0,96 & $-0,01$ & 0,94 \\
\hline IN080 & 0,12 & 0,88 & $-0,07$ & 0,79 \\
\hline IN085 & 0,3 & 0,82 & $-0,08$ & 0,77 \\
\hline IN007 & 0 & $-0,04$ & 0,89 & 0,79 \\
\hline IN035 & 0 & $-0,07$ & 0,78 & 0,62 \\
\hline IN036 & 0,05 & $-0,03$ & 0,97 & 0,94
\end{tabular}


(\%). Esses três indicadores representaram a dimensão operacional dos sistemas de abastecimento de água do banco de dados do SNIS. Assim, o fator 1 foi denominado Operacional.

Já o fator 2 foi formado pelos indicadores IN079 Índice de conformidade da quantidade de amostras - cloro residual (\%), IN080 Índice de conformidade da quantidade de amostras - turbidez (\%) e IN085 Índice de conformidade da quantidade de amostras — coliformes totais (\%), conforme pode ser observado pelo valor das cargas apresentadas na Tabela 2. Esses indicadores estão relacionados à qualidade da água fornecida à população, revelando se os operadores realizaram a quantidade mínima de análises relativas aos três parâmetros de potabilidade de água. Dessa forma, o fator 2 foi denominado Qualidade.

O fator 3, por sua vez, foi rotulado como Financeiro, visto que é formado pelos indicadores relativos à dimensão administrativafinanceira: IN007 Incidência da despesa de pessoal e de serviços de terceiros nas despesas totais com os serviços (\%), IN035 Participação da despesa com pessoal próprio nas despesas de exploração (\%) e IN036 Participação da despesa com pessoal total (equivalente) nas despesas de exploração (\%). Esse conjunto de indicadores representa a influência da despesa com pessoal nas despesas de exploração e total.

Os escores de cada fator foram gerados por meio do método de regressão, dando origem a um novo conjunto de índices que resumem as três dimensões do banco de dados do SNIS: Operacional, Qualidade e Financeiro. Os valores dos escores foram normalizados para que a escala desses índices fosse adimensional, variando de 0 a 1 . Assim, quanto mais próximo de 0 , pior é o desempenho do prestador; consequentemente, quanto mais próximo de 1, melhor é o seu desempenho.

\section{Comparação dos índices por meio de boxplots}

Foram gerados boxplots de modo a facilitar a visualização da evolução do desempenho dos prestadores públicos e privados entre os anos de 2003 e 2013 para cada um dos índices obtidos da análise fatorial. A interpretação dos índices considerou o sinal das cargas fatoriais, as quais revelam o relacionamento de cada variável (indicador do SNIS) com o valor do escore desse fator, conforme Tabela 2 .

A evolução do desempenho dos grupos público e privado para o índice Operacional está representada na Figura 1. Quanto mais próximo de 1, melhor é o desempenho operacional do prestador, refletindo os bons resultados para o índice de micromedição relativo ao volume disponibilizado e para o índice de faturamento de água. Em contrapartida, quanto mais próximo de 0 pior é o desempenho operacional, representando as altas porcentagens do índice de perdas de faturamento.

O grupo privado apresentou os maiores valores da mediana em todos os anos. No caso desse índice, dizer que o grupo privado teve o melhor desempenho operacional ao longo dos anos é também afirmar que esse grupo apresentou bons índices de faturamento e micromedição relativo ao volume disponibilizado, o que reforça as suspeitas de Scriptore e Toneto Júnior (2012) quanto ao melhor desempenho das empresas privadas nos aspectos técnicos. A principal diferença se concentrou em 2003, 2004 e 2005, apresentando um comportamento atípico dos demais em relação à dispersão dos dados. Tal comportamento deve ser investigado a fim de elucidar as possíveis causas, principalmente em relação à qualidade dos dados.

A mesma análise foi realizada para os grupos público e privado, discriminando, dessa vez, a Sanepar. Dessa forma, ficou evidente que a Sanepar apresentava melhor desempenho em relação aos demais

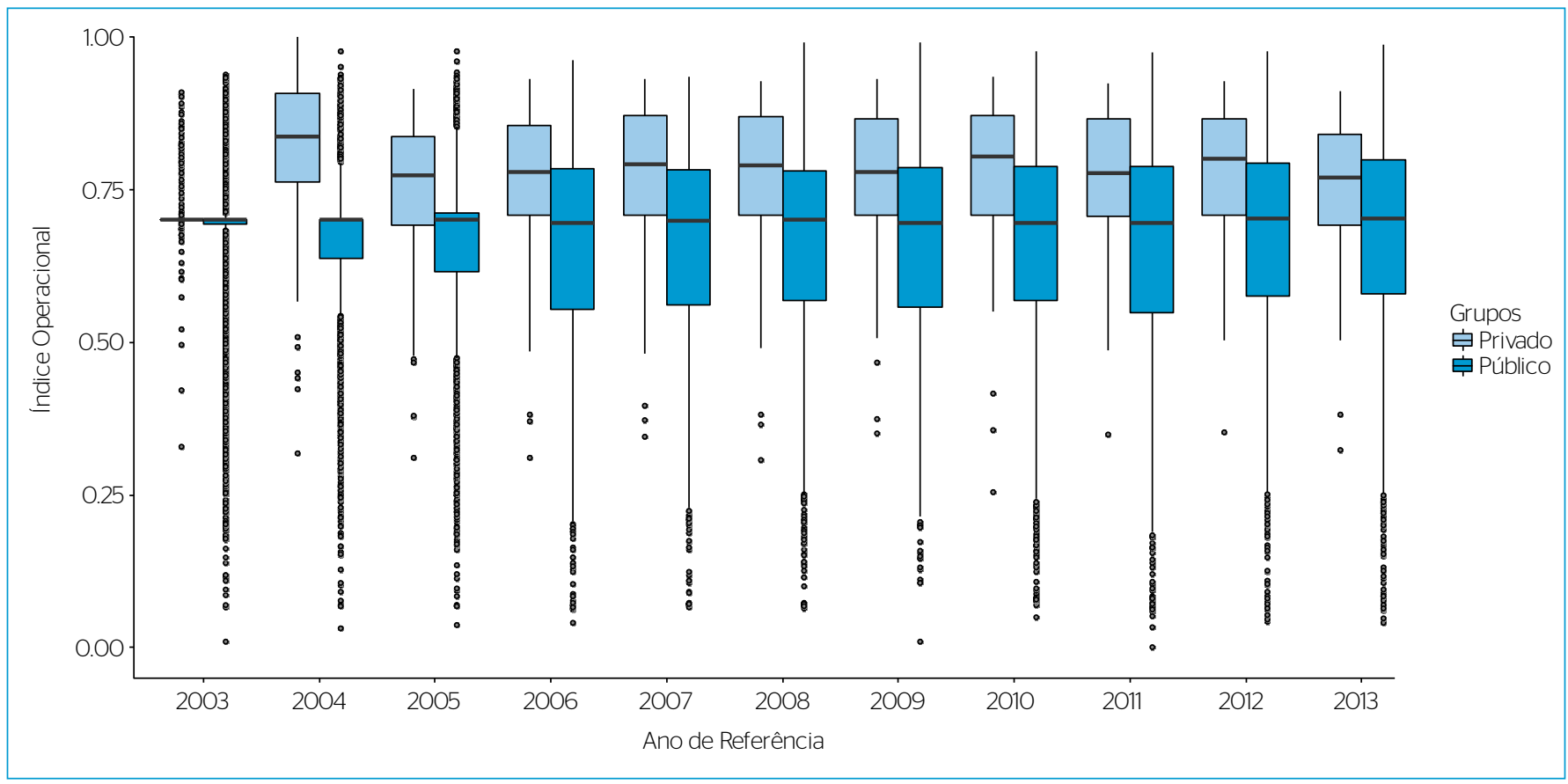

Figura 1 - Boxplot da evolução do índice Operacional. 
grupos em cada ano, conforme Figura 2. Outro ponto interessante foi o desempenho das empresas privadas, que, na maioria dos anos, tiveram valores da mediana inferiores aos do grupo público.

As Figuras 3 e 4 apresentam a evolução do índice de Qualidade obtido por meio dos escores do fator 2, considerando as duas situações de análise. Os boxplots demonstraram que tanto o grupo público quanto o grupo privado apresentaram comportamentos parecidos, sendo que a principal diferença se confere pela dispersão dos dados. No entanto, o valor das medianas seguiu uma mesma tendência: os dois grupos possuem valores próximos.

Ressalta-se que os dados para 2003, 2004, 2005 e 2013 apresentaram comportamento atípico, principalmente em relação à dispersão. A principal diferença desses anos para os demais foi a quantidade de dados ausentes que foram substituídos pela média durante o cálculo

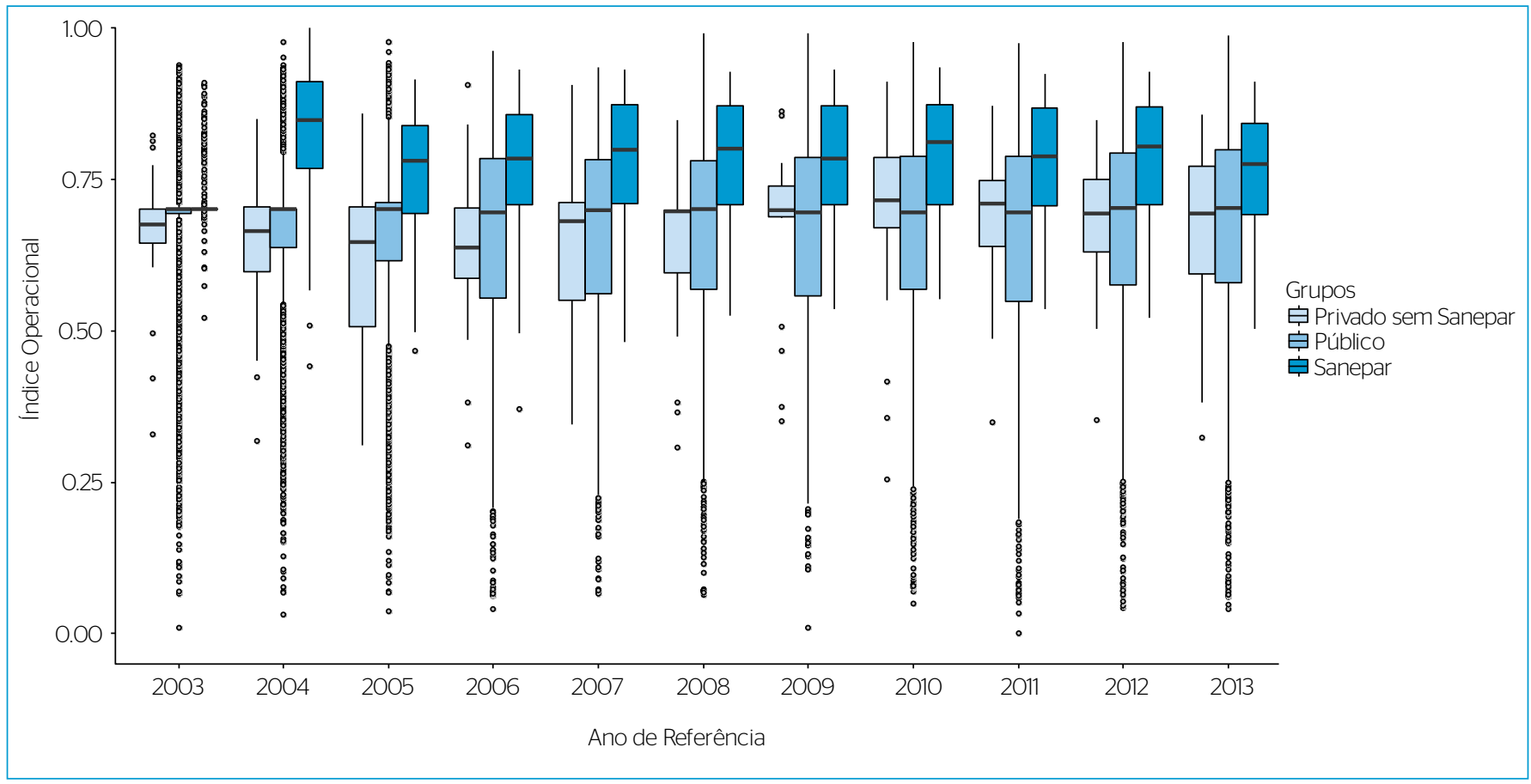

Figura 2 - Boxplots da evolução do índice Operacional para os três grupos.

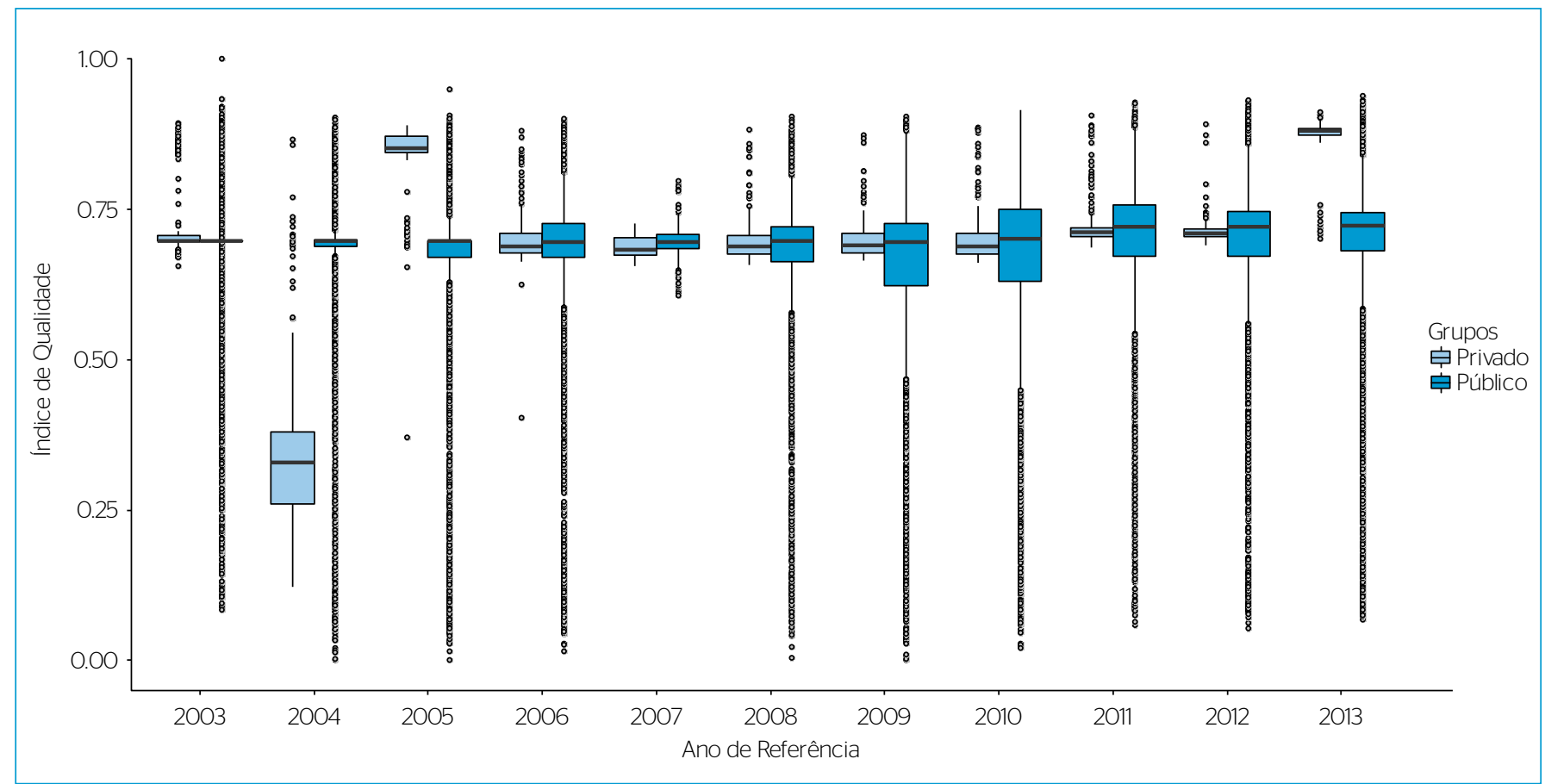

Figura 3 - Boxplots da evolução do índice de Qualidade. 
dos escores, o que explicaria a diferença na dispersão dos dados para os demais anos. A baixa qualidade dos dados do SNIS sempre é ressaltada como uma das principais dificuldades e ressalvas de trabalhos já realizados baseados nesse banco de dados (FONSECA \& GABRIEL, 2015).

Com exceção dos anos mencionados anteriormente, pôde-se perceber que não há diferença entre os grupos tanto da primeira análise (público e privado) quanto da segunda análise (público, privado sem
Sanepar e Sanepar). Ou seja, a gestão privada não diferenciaria os prestadores em relação ao desempenho no cumprimento das análises obrigatórias para garantir o padrão de potabilidade da água.

Em relação às comparações do índice Financeiro entre os grupos público e privado (ver Figura 5), este apresentou os melhores resultados, exceto em 2003. Observou-se que, no último ano, o valor da mediana de ambos foi de 0,38 , o que revelou um baixo desempenho dos prestadores em

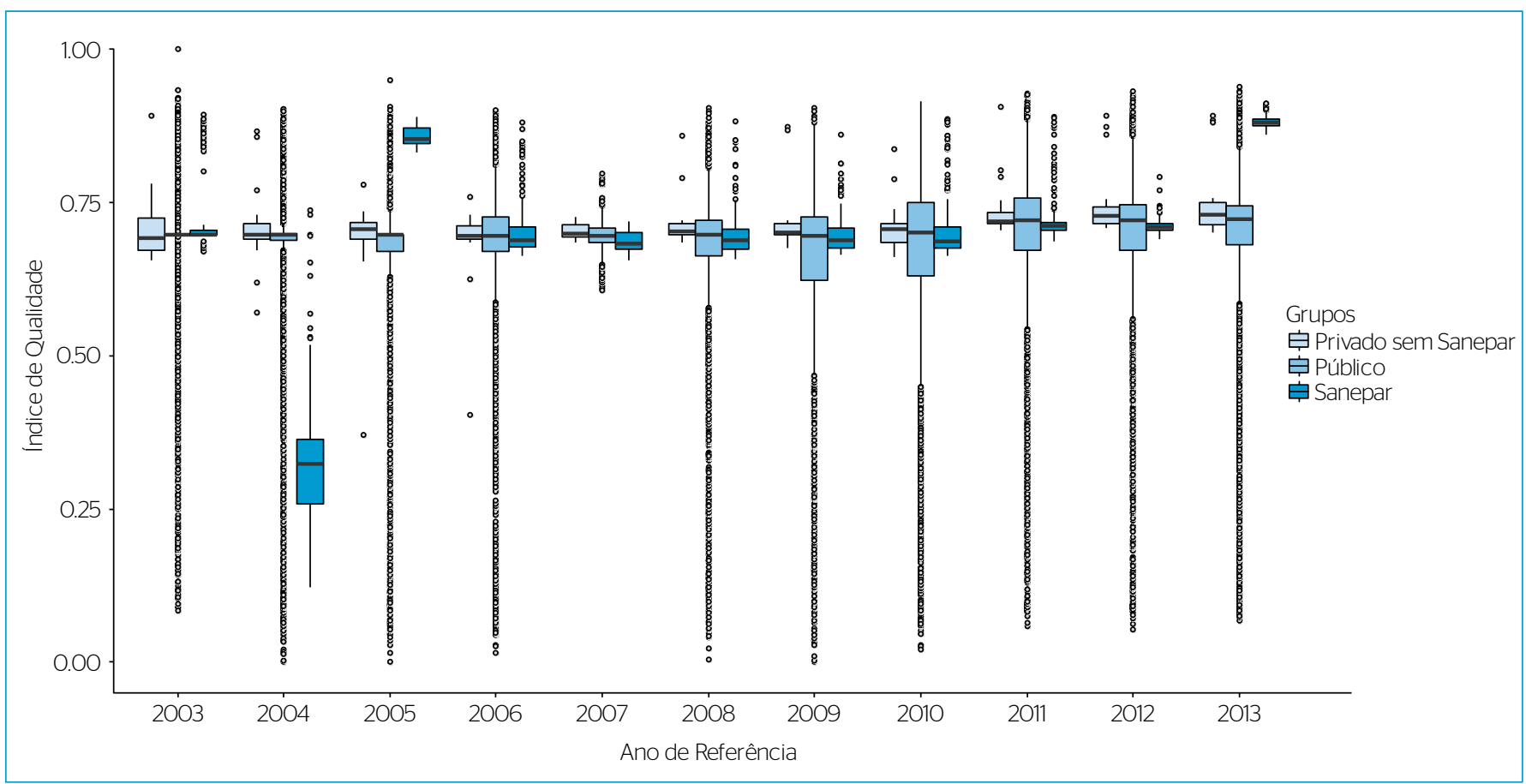

Figura 4 - Boxplots da evolução do índice de Qualidade para os três grupos.

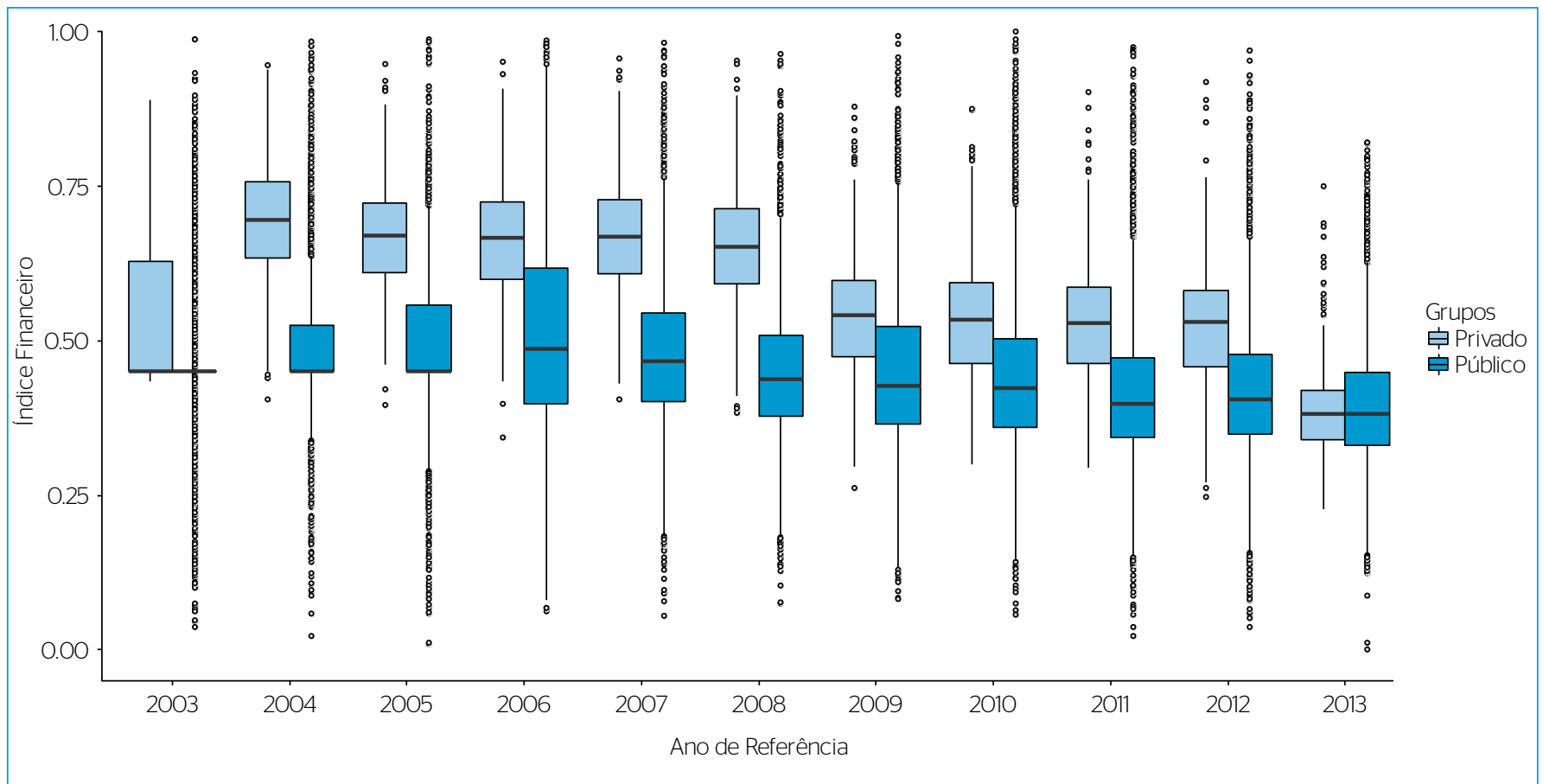

Figura 5 - Boxplots da evolução do índice Financeiro. 
relação à influência nas despesas com pessoal. Nesse índice, esperava-se que o grupo público tivesse valores mais próximos a 0 em razão da suposição do excessivo quadro de pessoal devido às interferências políticas (PARLATORE, 2000).

Ao considerar os três grupos (público, privado e Sanepar), conforme Figura 6, não se observou clareza em relação ao melhor desempenho, que pareceu oscilar entre as empresas privadas e a Sanepar.

Comparação dos índices por meio dos testes de Wilcoxon-MannWhitney e de Kruskall-Wallis

Por meio do teste de Wilcoxon-Mann-Whitney, comparou-se os grupos público e privado. De uma forma geral, a $\mathrm{H} 0$ foi a de que o desempenho para os índices Operacional, de Qualidade e Financeiro não difere significativamente entre os grupos público e privado, ao contrário da hipótese alternativa $(\mathrm{H} 1)$, que relata diferença entre os grupos, no que se refere a esses índices.

Os resultados para esse teste estão apresentados por ano por meio dos valores da estatística de Wilcoxon-Mann-Whitney e do valor p para cada índice, conforme Tabela 3. A H0 foi rejeitada nos casos em que o valor $\mathrm{p}$ foi menor ou igual ao nível de significância de 5\%.

Em relação ao índice Operacional, a H0 foi rejeitada em todos os anos, visto que o valor $p$ foi inferior a 0,05 . A rejeição dessa hipótese, junto à análise da estatística descritiva, corrobora com as expectativas favoráveis à privatização quanto à melhor performance nos aspectos operacionais dos serviços de água, o que apoia a ideia de que os operadores privados se destacam na capacidade técnica (SCRIPTORE \& TONETO JÚNIOR, 2012; ABCON \& SIDCON, 2015).
Tabela 3 - Resultados do teste de Wilcoxon-Mann-Whitney para os índices Operacional, de Qualidade e Financeiro.

\begin{tabular}{|c|c|c|c|c|}
\hline Ano & $\begin{array}{c}\text { Teste de } \\
\text { Wilcoxon-Mann- } \\
\text { Whitney }\end{array}$ & $\begin{array}{c}\text { Índice } \\
\text { Operacional }\end{array}$ & $\begin{array}{l}\text { Índice de } \\
\text { Qualidade }\end{array}$ & $\begin{array}{c}\text { Indice } \\
\text { Financeiro }\end{array}$ \\
\hline \multirow{2}{*}{2003} & W & 716488,5 & 703014,5 & 443581,5 \\
\hline & Valor $p$ & O & O & O \\
\hline \multirow{2}{*}{2004} & W & 1049301 & 111131 & 151140 \\
\hline & Valor $p$ & 0 & 0 & O \\
\hline \multirow{2}{*}{2005} & W & 798872 & 1162658 & 169594 \\
\hline & Valor p & 0 & 0 & O \\
\hline \multirow{2}{*}{2006} & W & 857121 & 562060 & 237365 \\
\hline & Valor $p$ & O & 0 & O \\
\hline \multirow{2}{*}{2007} & W & 895521 & 418589 & 136173 \\
\hline & Valor $p$ & O & 0 & O \\
\hline \multirow{2}{*}{2008} & W & 880258 & 549390 & 108253 \\
\hline & Valor p & 0 & 0 & 0 \\
\hline \multirow{2}{*}{2009} & W & 882639 & 592430 & 334719 \\
\hline & Valor p & 0 & 0,59 & O \\
\hline \multirow{2}{*}{2010} & W & 895241 & 565801 & 319611 \\
\hline & Valor p & 0 & 0,06 & O \\
\hline \multirow{2}{*}{2011} & W & 872730 & 299243 & 253987 \\
\hline & Valor $p$ & O & 0 & O \\
\hline \multirow{2}{*}{2012} & W & 875004 & 494464 & 281888 \\
\hline & Valor $p$ & 0 & O & 0 \\
\hline \multirow{2}{*}{2013} & W & 788168 & 1134282 & 616701 \\
\hline & Valor $p$ & 0 & 0 & 0,47 \\
\hline
\end{tabular}

W: teste de Wilcoxon-Mann-Whitney

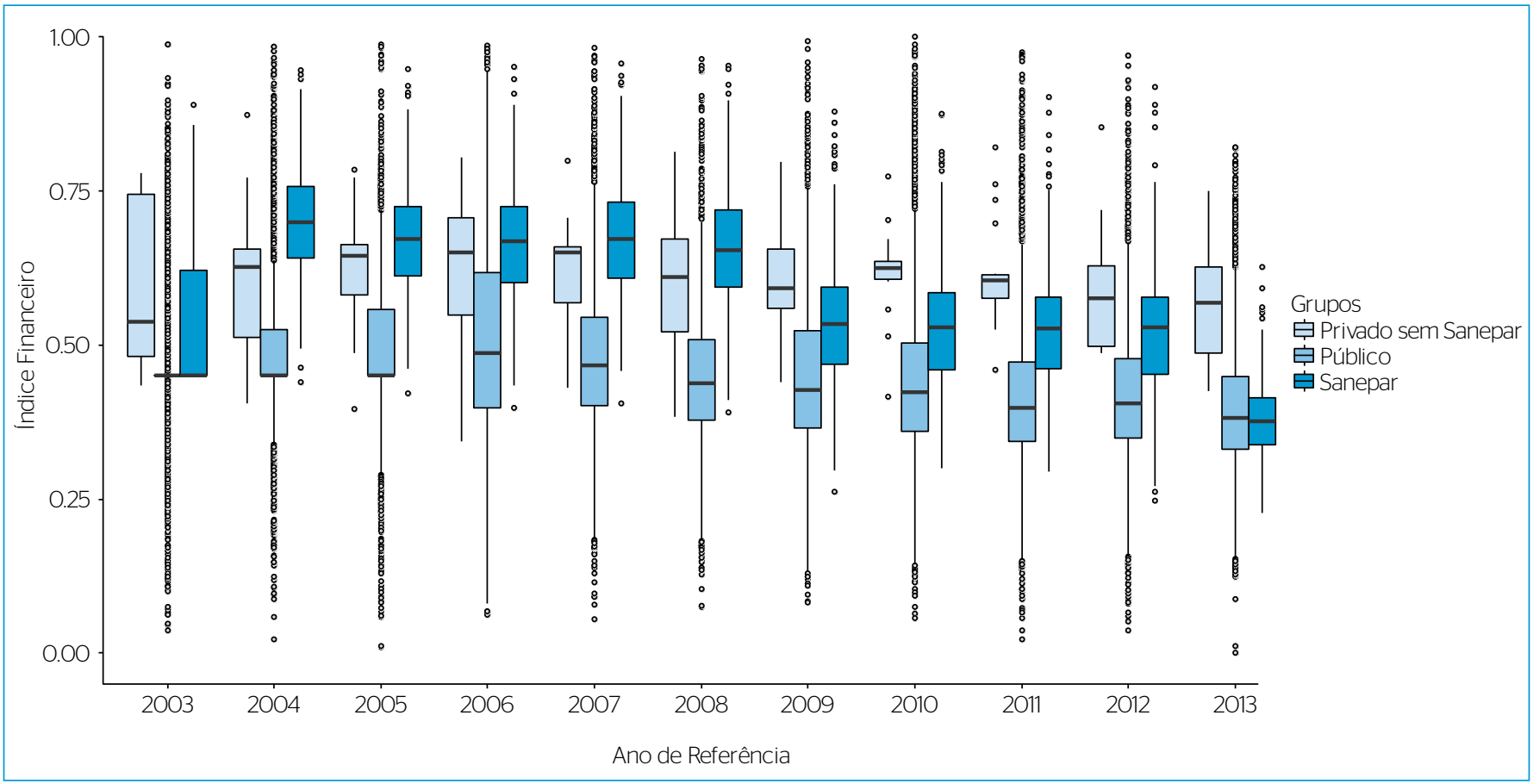

Figura 6 - Boxplots da evolução do índice Financeiro para os três grupos. 
Apesar de a análise visual da Figura 3 sugerir que os grupos público e privado possuem comportamento da mediana similar, o resultado do teste de Wilcoxon-Mann-Whitney revelou que há diferença significativa em relação ao cumprimento das análises obrigatórias dos três parâmetros de potabilidade da água - assim como os resultados do teste para o índice Financeiro sugeriram diferença significativa entre os grupos em relação à influência da despesa com pessoal nas despesas totais e nas despesas de exploração.
Os resultados do teste de Kruskal-Wallis e de comparações múltiplas para os grupos público, privado e Sanepar também sugeriram diferença significativa. A Tabela 4 apresenta os valores de p do teste de KruskalWallis, ano a ano, para cada índice e, em seguida, os resultados do teste de comparações múltiplas, indicando se houve diferença entre os grupos comparados em pares. Rejeitou-se a $\mathrm{H} 0$ quando o valor $\mathrm{p}$ foi igual ou inferior a 0,05 . Observa-se que o valor $\mathrm{p}$ foi inferior a $0,05 \mathrm{em}$

Tabela 4 - Resultados do teste de Kruskal-Wallis e do teste de comparações múltiplas.

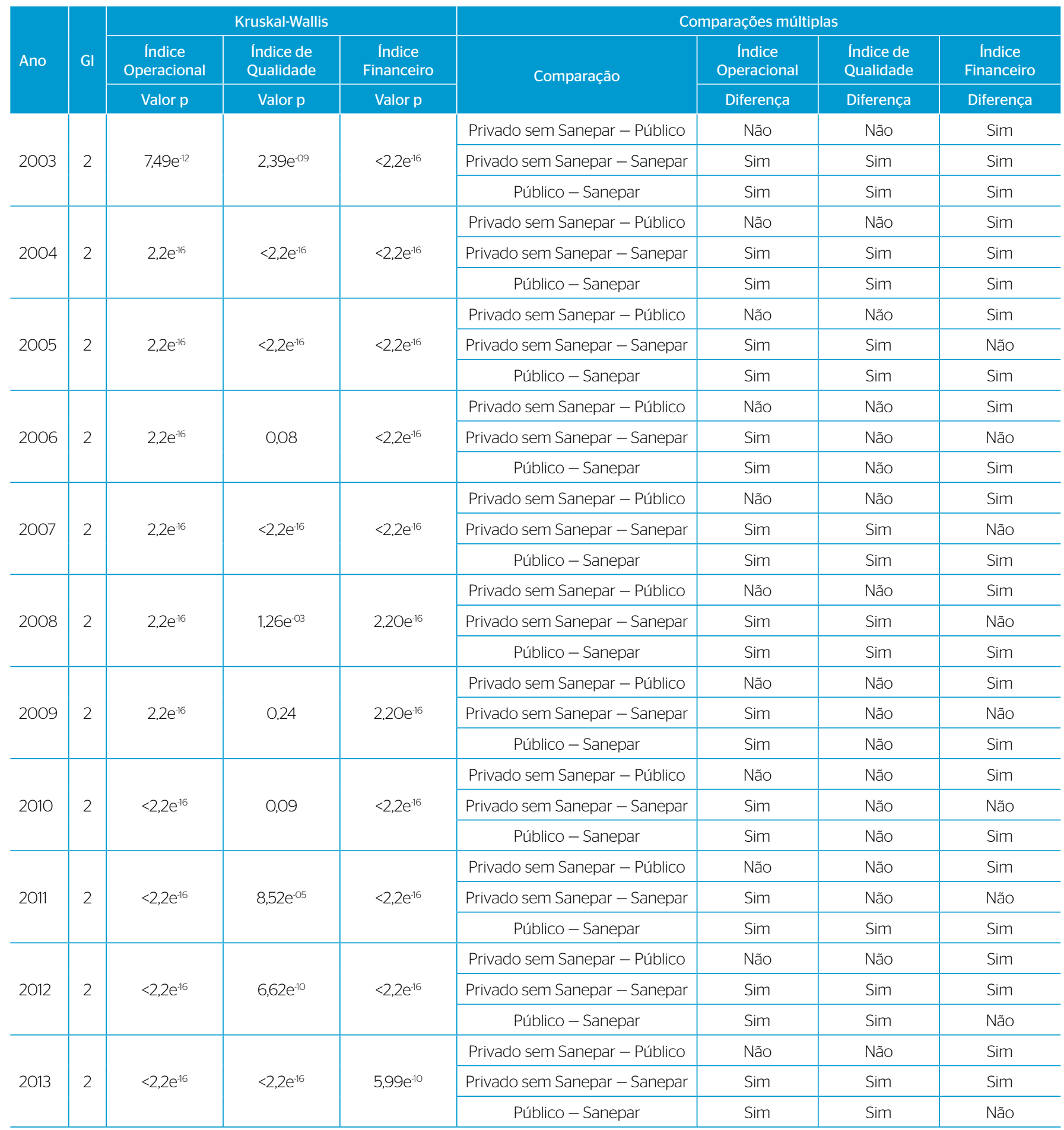


todos os índices para todos os anos, conforme Tabela 4. Dessa forma, rejeitou-se a $\mathrm{H} 0$, de que não há diferença entre os grupos, e aceitou-se a $\mathrm{H} 1$ de que há diferença significativa em pelo menos dois grupos. Em seguida, o teste de comparações múltiplas permitiu identificar os grupos que divergiram entre eles.

No caso do índice Operacional, confirmou-se a suspeita de diferença entre a performance da Sanepar e dos demais grupos em todos os anos. Além disso, o desempenho dos grupos público e privado sem Sanepar para o índice Operacional é igual estatisticamente.

Em relação ao índice de Qualidade, os resultados foram semelhantes aos do índice Operacional. Não há diferença estatisticamente significativa no cumprimento das análises obrigatórias dos parâmetros de potabilidade de água entre os grupos público e privado sem Sanepar. No entanto, os valores da mediana da Sanepar para esse índice divergiram significativamente na maioria dos anos com todos os demais grupos, porém houve algumas exceções em 2006, 2009, 2010 e 2011. Diferentemente dos índices anteriores, houve diferença significativa no desempenho do índice Financeiro entre os grupos público e privado sem Sanepar em todos os anos, enquanto que na maioria dos anos não houve diferença significativa nos valores das medianas entre a Sanepar e os outros grupos.

Portanto, os resultados do teste de Wilcoxon-Mann-Whitney corroboram o argumento de que existem diferenças significativas entre os indicadores operacionais, financeiros e de qualidade dos serviços de água prestados para cada ano. Por outro lado, o teste de Kruskal-Wallis e as comparações múltiplas indicaram que o desempenho da Sanepar foi o que mais se diferenciou dos demais grupos.

\section{Análise de regressão linear múltipla}

Houve a violação da normalidade e da homocedasticidade dos resíduos em todos os modelos ajustados, o que inviabilizou qualquer análise a partir dos seus parâmetros estimados. Além disso, o valor do coeficiente de determinação de todos os modelos ficou abaixo de 0,15 , ou seja, menos de $15 \%$ da variabilidade dos dados foi explicado pelos modelos ajustados - o mínimo recomendado em literatura é 60\%. Portanto, não houve interpretação dos parâmetros estimados, a fim de evitar análises equivocadas. Esse resultado evidencia uma limitação dos modelos tradicionais de análise de regressão linear múltipla para os dados do SNIS e do IBGE.

\section{CONSIDERAÇÕES FINAIS}

A comparação realizada neste trabalho sugeriu que há diferença de desempenho na prestação de serviços de abastecimento de água entre grupos. $\mathrm{O}$ grupo de prestadores privatizados tendeu a manter melhores índices de desempenho ano a ano, desde o início do período analisado. A Sanepar influenciou o desempenho do grupo privado e destacou-se na maioria dos índices. No entanto, vale ressaltar que os prestadores públicos e privados partiram de situações distintas, visto que os municípios com serviços privatizados já apresentavam uma situação melhor do que aqueles com prestadores públicos em 2003.

Para gerar evidências mais conclusivas sobre o tema, recomenda-se definir e analisar a evolução de uma amostra de prestadores públicos e privados que tenham no início da série histórica o mesmo nível de desempenho e, a partir daí, analisar a influência do tipo de gestão na evolução da performance dos prestadores. Uma outra possibilidade seria fazer o ajuste de um modelo estatístico longitudinal, mais elaborado, que levasse em conta todas as variáveis sobre as condições do município que pudessem influenciar nos indicadores.

Destacamos, finalmente, que a influência da participação privada nos serviços de saneamento pode ser analisada por meio de diversas abordagens metodológicas. Este estudo se limitou à análise quantitativa dos dados secundários do SNIS; todavia, espera-se que a academia continue a pesquisar esse tema, por exemplo, por meio de estudos de caso (únicos e múltiplos), nos quais o desempenho seja avaliado a partir de dados e depoimentos coletados diretamente nos prestadores de serviços.

\section{REFERÊNCIAS}

ALENCAR FILHO, F.M. de; ABREU, L.M. de. (2005) Metodologia alternativa para avaliação de desempenho de companhias de saneamento básico: aplicação da análise fatorial. Planejamento e Políticas Públicas, n. 28, p. 23-39.

ARARAL, E. (2009) The failure of water utilities privatization: Synthesis of evidence, analysis and implications. Policy and Society, v. 27, n. 3, p. 221-228. https://doi.org/10.1016/j. polsoc.2008.10.006
ASSOCIAÇÃO BRASILEIRA DE CONCESSIONÁRIAS PRIVADAS DE SERVIÇOS PÚBLICOS DE ÁGUA E ESGOTO (ABCON); SINDICATO NACIONAL DAS CONCESSIONÁRIAS PRIVADAS DE SERVIÇOS PÚBLICOS DE ÁGUA E ESGOTO (SINDCON). (2015) Panorama da Participação Privada no Saneamento Brasil. ABCON/SINDCON. 79 p.

BAER, M. (2014) Private Water, Public Good: Water Privatizationand State Capacity in Chile. Studies in Comparative International Development, v. 49, n. 2, p. 141-167. https://doi.org/10.1007/s12116-014-9154-2 
BEZERRA, F.A.; CORRAR, L.J. (2006) Utilização da análise fatorial na identificação dos principais indicadores para avaliação do desempenho financeiro: uma aplicação nas empresas de seguros. Revista Contabilidade \& Finanças, v. 17 , n. 42 , p. 50-62. http://dx.doi.org/10.1590/S151970772006000300005

BOLLMANN, H.A.; MARQUES, D.D.M. (2000) Bases para a estruturação de indicadores de qualidade de águas. Revista Brasileira de Recursos Hídricos, v. 5, n. 1, p. 37-60. http://dx.doi. org/10.21168/rbrh.v5n1.p37-60

BORRAZ, F; PAMPILLON, N.G.; OLARREAGA, M. (2013) Water nationalization and service quality. World Bank Economic Review, v. 27, n. 3, p. 389-412.

BRASIL. Ministério das Cidades. Secretaria Nacional de Saneamento. (2014) Sistema Nacional de Informações sobre Saneamento: Diagnóstico dos Serviços de Água e Esgotos 2013. Brasília: Secretaria Nacional de Saneamento/Ministério das Cidades.

BRASIL. Ministério das Cidades. Secretaria Nacional de Saneamento. Sistema Nacional de Informações sobre Saneamento. Aplicativo Série Histórica. Disponível em: <http://app. cidades.gov.br/serieHistorica/>. Acesso em: 2 fev. 2015.

BUDDS, J.; MCGRANAHAN, G. (2003) Are the debates on water privatization missing the point? Experiences from Africa, Asia and Latin America. Environment\&Urbanization, v. 15, n. 2, p. 87-114. https://doi.org/10.1177/09562478030150022

COSTELLO, A.B.; OSBORNE, J.W. (2005) Best Practices in Exploratory Factor Analysis: Four Recommendations for Getting the Most From Your Analysis. Pratical Assessment, Research \& Evaluation, v. 10, n. 7. p. 27-29.

DA SILVA E SOUZA, G.; DE FARIA, R.C.; MOREIRA, T.B.S. (2007) Estimating the relative efficiency of Brazilian publicly and privately owned water utilities: A stochastic cost frontier approach. Journal of the American Water Resources Association, v. 43, n. 5, p. 1237-1244. https://doi.org/10.1111/j.17521688.2007.00106.x

DRAPER, N.R.; SMITH, H. (1998) Applied Regression Analysis. 3. ed. Nova York: John Wiley.

FARIA, R.C.; FARIA, S.A.; MOREIRA, T.B.S. (2005) A privatização no setor de saneamento tem melhorado a performance dos serviços ? Planejamento e Políticas Públicas, n. 28, p. 7-21.

FERRO, G.; LENTINI, E.J.; MERCADIER, A.C.; ROMERO, C.A. (2014) Efficiency in Brazil's water and sanitation sector and its relationship with regional provision, property and the independence of operators. Utilities Policy, v. 28, p. 42-51. https:// doi.org/10.1016/j.jup.2013.12.001

FIGUEIRÊDO, M.C.B.; ROSA, M.F.; MATTOS, A.L.A.; MOTA, S. (2010) Avaliação do desempenho ambiental de inovações tecnológicas agroindustriais: conceitos e métodos. Fortaleza: Embrapa Agroindústria Tropical. 44 p.
FIGUEIREDO FILHO, D.; NUNES, F.; ROCHA, E.C. da; SANTOS, M.L.; BATISTA, M.; SILVA JÚNIOR, J.A. (2O11) O que fazer e o que não fazer com a regressão: Pressupostos e aplicações do modelo linear de mínimos quadrados ordinários (MQO). Revista Política Hoje, v. 20, n. 1, p. 44-99.

FIGUEIREDO FILHO, D.B.; SILVA JÚNIOR, J.A. da. (2010) Visão além do alcance: uma introdução à análise fatorial. Opinião Pública, v. 16, n. 1, p. 160-185. http://dx.doi.org/10.1590/S010462762010000100007

FONSECA, A.; GABRIEL, C.F. (2015) Análise da influência da tarifação em seis indicadores operacionais e de qualidade dos serviços de abastecimento de água no Brasil. Engenharia Sanitaria e Ambiental, v. 20, n. 2, p. 219-224. http://dx.doi.org/10.1590/S141341522015020000129123

FOSTER, V. (2005) Ten years of water service reform in Latin America: Toward an Anglo-French model. Washington, D.C.: World Bank. (Water Supply and Sanitation Sector Board Discussion, n. 3).

HAILU, D.; OSORIO, R.G.; TSUKADA, R. (2012) Privatization and Renationalization: What Went Wrong in Bolivia's Water Sector? World Development, v. 40, n. 12, p. 2564-2577. http://dx.doi. org/10.1016/j.worlddev.2012.05.032

HAIR, J.F.; BLACK, W.C.; BABIN, B.J.; ANDERSON, R.E.; TATHAM, R.L. (2006) Análise fatorial. In: HAIR, J.F.; BLACK, W.C.; BABIN, B.J.; ANDERSON, R.E.; TATHAM, R.L. Análise multivariada de dados. 6. ed. Porto Alegre: Bookman. p. 100-146.

HARMAN, H.; JONES, W.H. (1966) Factor analysis by minimizing residuals (minres). Psychometrika, v. 31, n. 3, p. 351-368.

HELLER, P.G.B.; HELLER, B.; HELLER, L. (2009) Desempenho tecnológico dos serviços de abastecimento deágua e esgotamento sanitário em quatro municípios de Minas Gerais: uma análise comparativa. Revista Engenharia Sanitária Ambiental, v. 14, n. 1, p. 109-118. http://dx.doi.org/10.1590/S1413-41522009000100012

HELLER, P.G.B.; NASCIMENTO, N.O.; HELLER, L.; MINGOTI, S.A. (2O12) Desempenho dos diferentes modelos institucionais de prestação dos serviços públicos de abastecimento de água : uma avaliação comparativa no conjunto dos municípios brasileiros. Engenharia Sanitaria e Ambiental, v. 17, n. 3, p. 333-342. http://dx.doi.org/10.1590/ S1413-41522012000300010

OLIVEIRA, T.G.; REZENDE, S.; HELLER, L. (2011) Privatização dos serviços de saneamento: uma análise qualitativa à luz do caso de Cachoeiro de Itapemirim (ES). Revista Engenharia Sanitária e Ambiental, v. 16, n. 4, p. 395-402. http://dx.doi.org/101590/S141341522011000400011

OUDA, O.K.M.; AL-WAKED, R.F.; ALSHEHRI, A.A. (2014) Privatization of water-supply services in Saudi Arabia: A unique experience. Utilities Policy, v. 31, p. 107-113. https://doi.org/10.1016/j.jup.2014.10.003

PALUDO, J.R.; BORBA, J. (2013) Abastecimento de água e esgotamento sanitário: estudo comparado de modelos de gestão em Santa Catarina. Ambiente \& Sociedade, v. 16, n. 1, p. 59-78. http:// dx.doi.org/10.1590/S1414-753X2013000100005 
PARLATORE, A.C. (2000) Privatização do setor de saneamento no Brasil. In: PINHEIRO, A.C.; FUKASAKU, K. A privatização no Brasil: o caso dos serviços de utilidade pública. Rio de Janeiro: BNDES. p. 281-320.

PÉRARD, E. (2009) Water supply: Public or private? Policy and Society, v. 27, n. 3, p. 193-219. https://doi.org/10.1016/j. polsoc.2008.10.004

REVELLE, W. Psych: Procedures for Personality and Psychological Research. Illinois: Northwestern University. Disponível em: <https:/l www.scholars.northwestern.edu/en/publications/psych-proceduresfor-personality-and-psychological-research>. Acesso em: 3 ago. 2015.

REZENDE, M.L.; FERNANDES, L.P.D.S.; SILVA, A.M.R.E. (2007) Utilização da análise fatorial para determinar o potencial de crescimento econômico em uma região do Sudeste do Brasil. Revista Economia e Desenvo/vimento, v. 19, p. 92-109.

SABBIONI, G. (2008) Efficiency in the Brazilian sanitation sector. Utilities Policy, v. 16, n. 1, p. 11-20. https://doi.org/10.1016/j.jup.2007.06.003

SAIANI, C.C.S.; TONETO JÚNIOR, R. (2010) Evolução do acesso a serviços de saneamento básico no Brasil (1970 a 2004). Economia e Sociedade, v. 19, n. 1, p. 79-106.
SCRIPTORE, J.S; TONETO JÚNIOR, R. (2O12) A estrutura de provisão dos serviços de saneamento básico no Brasil: uma análise comparativa do desempenho dos provedores públicos e privados. Revista de Administração Pública, v. 46, n. 6. p. 1479-1504. http://dx.doi. org/10.1590/S0034-76122012000600004

SILVEIRA, B.C:; SILVA, R.G. da; CARVALHO, L. de A. (2008) Indice relativo de qualidade de vida da Região Norte: uma aplicação da análise fatorial. Revista Brasileira de Gestão e Desenvolvimento Regional, v. 4, n. 4, p. 80-97.

TUPPER, H.C.; RESENDE, M. (2004) Efficiency and regulatory issues in the Brazilian water and sewage sector: an empirical study. Utilities Policy, v. 12, n. 1, p. 29-40. https://doi.org/10.1016/j. jup.2003.11.001

VARGAS, M.C:; GOUVELLO, B. de. (2011) Trajetória e perspectivas da gestão privada do saneamento na América Latina: contrastes e aproximações entre Brasil e Argentina. Desenvo/vimento e Meio Ambiente, n. 24, p. 57-70.

ZAKI, S.; NURUL AMIN, A.T.M. (2009) Does Basic Services Privatisation Benefit the Urban Poor? Some Evidence from Water Supply Privatisation in Thailand. Urban Studies, v. 46, n. 11, p. 2301-2327. https://doi.org/10.1177/0042098009342902 